\title{
Cutaneous cytomegalovirus infection in a child with hyper IgE and specific defects in antibody response to protein vaccines
}

Authors

Shahrzad Fallah ${ }^{1}$

Ahmad Tabatabaei

Zahra Pournasir ${ }^{2}$

Zahra Chavoshzadeh ${ }^{3}$

Nima Rezaei ${ }^{4}$

${ }^{1}$ Department of Pediatrics, Mofid Children Hospital, Shaheed Beheshti

University of Medical

Sciences, Tehran, Iran

${ }^{2}$ Loghman Hospital,

Shaheed Beheshti

University of Medical

Sciences, Tehran, Iran

${ }^{3}$ Pediatric Infectious

Research Center, Shaheed Beheshti University of

Medical Sciences,

Tehran, Iran

${ }^{4}$ Research Center for

Immunodeficiencies,

Children's Medical

Center, and Molecular

Immunology Research

Center; Department of

Immunology, School

of Medicine, Tehran

University of Medical

Sciences, Tehran, Iran

Submitted on: 02/13/2011 Approved on: 03/09/2011

Correspondence to: Zahra Chavoshzadeh Pediatric Infections Research Center Mofid Children Hospital Shaheed Beheshti Medical University

14194, Tehran, Iran zahra_chavoshzadeh@ yahoo.com

We declare no conflict of interest.

๑2011 Elsevier Editora Ltda. All rights reserved.

\begin{abstract}
Cytomegalovirus (CMV) infection is a common opportunistic systemic infection in immunocompromised patients, but skin involvement is rare. Herein, we report a 10 year-old girl from consanguineous parents who was referred to our center because of disseminated maculopapular rash. She had history of upper and lower respiratory tract infections. In immunological studies, increased serum IgE level and decreased responses to tetanus and diphtheria were detected. Polymerase chain reaction (PCR) examination of bronchoalveolar lavage and serum sample revealed the presence of CMV. Early diagnosis of cutaneous CMV and appropriate treatment are the key actions in management of patients with underlying immunodeficiencies to avoid further complications.
\end{abstract}

Keywords: cytomegalovirus infections; antibody formation; common variable immunodeficiency.

\section{CASE REPORT}

Cytomegalovirus (CMV) is a DNA virus that causes various dermatologic and systemic disorders, especially in immunocompromised subjects. ${ }^{1}$ Cutaneous manifestation of CMV is a rare condition that its diagnosis is often made with delay. ${ }^{2}$

Herein, a 10 year-old girl from consanguineous parents was admitted to our center (Tehran, Iran) with complaint of disseminated maculopapular rash. It started as local macular rash one year before that gradually spread to other regions. She had history of recurrent otitis media and recurrent respiratory tract infections from early infancy. She also suffered from cervical lymphadenopathy.

There was generalized erythematous maculopapular rash involving all her body, especially on the limbs (Figure 1). Light microscopy examination of skin revealed patchy lymphocytic infiltration.

Complete blood cells and serum levels of IgA, IgM, IgG, and IgG subclasses as well as $\mathrm{C} 3, \mathrm{C} 4$ and $\mathrm{CH} 50$ were within normal range. However, serum IgE level was $482.7 \mathrm{IU}$, which was much higher than the upper limit of normal range (0-127 IU).

Flow cytometry study for lymphocyte subpopulations showed normal B- and T- cell counts. Isohaemagglutinin test and antibody

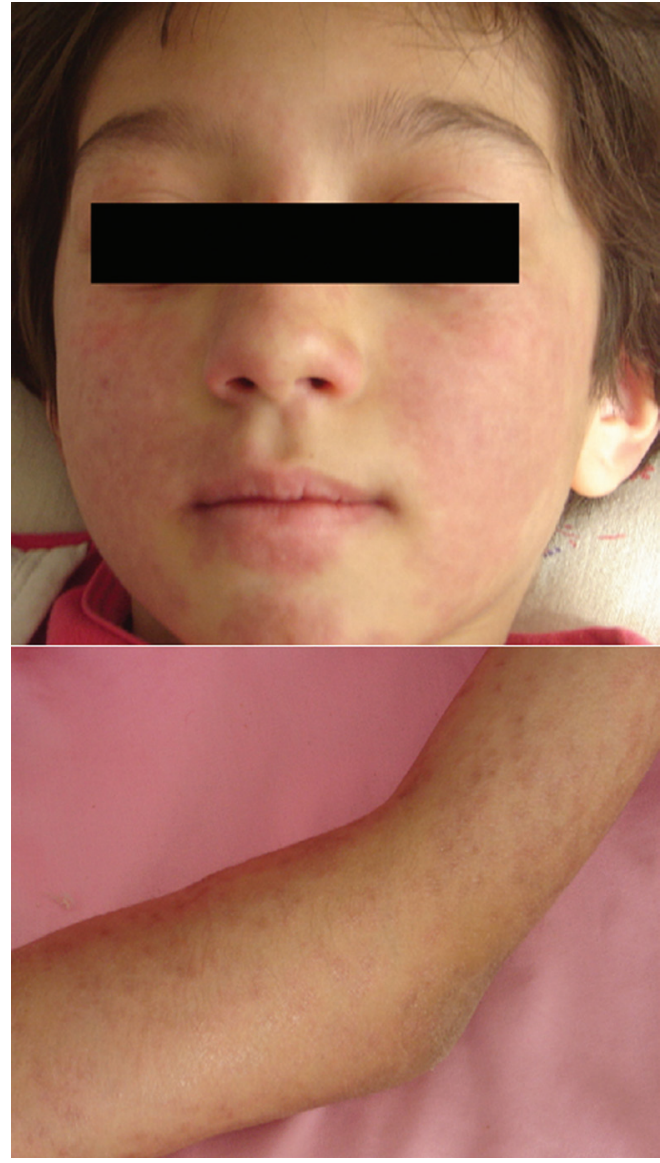

Figure 1: Generalized erythematous maculopapular rash of the patient. 
response to pneumococcus vaccine was normal. However, responses to tetanus and diphtheria were decreased $(<0.1$ and $<0.1 \mathrm{IU} / \mathrm{mL}$, respectively). $\mathrm{T}$ lymphocyte response to mitogens and lymphoblastic transformation test with phytohemagglutinin (PHA) and Candida antigens were normal.

Other laboratory tests were negative. HIV Ab test was negative. Bone marrow immunophenotyping was normal.

Chest computed tomography scan showed central bronchiectatic changes with prebronchial infiltration. Bronchoscopy with BAL was performed. Cytologic evaluation of bronchoalveolar lavage (BAL) showed acute inflammatory process. Polymerase chain reaction (PCR) examination of BAL sample revealed the presence of CMV. Qualitative PCR examination of blood, urine, and skin biopsy was highly positive for CMV.

Intravenous gancyclovir $10 \mathrm{mg} / \mathrm{kg} /$ day was administered for two weeks followed by gancyclovir $5 \mathrm{mg} / \mathrm{kg} /$ day for three weeks. Antibiotic therapy and intravenous immunoglobulin were also started for the patient during hospitalization. Skin eruption got better and patient was discharged from the hospital.

CMV infection in combined immunodeficiency is common; however, presence of cutaneous CMV in combined immunodeficiency is unusual. The presented patient had slightly increased level of IgE, but she had not all criteria for hyper IgE syndrome (HIES). Indeed she had some evidences of combined immunodeficiency, which is unusual in autosomal dominant form of HIES due to STAT3 (signal transducer and activator of transcription 3) mutation, but common in autosomal recessive form of HIES due to DOCK8 (dedicator of cytokinesis 8 ) mutation. ${ }^{3}$ Surprisingly, patients with in autosomal recessive HIES suffer from eczema and recurrent cutaneous infections ${ }^{3}$ and severe viral infections have been frequently seen in DOCK8 deficiency. ${ }^{4}$

It should be emphasized that early diagnosis of cutaneous CMV in patients with underlying immunodeficiencies and appropriate treatment are essential to avoid dissemination of the disease, further complications and even death in such patients. However, further molecular studies are needed to make definite diagnosis in such cases.

\section{REFERENCES}

1. Drago F, Aragone MG, Lugani C et al. Cytomegalovirus infection in normal and immunocompromised humans. A review. Dermatology 2000; 200:189-95.

2. Colsky AS, Jegasothy SM, Leonardi C et al. Diagnosis and treatment of a case of cutaneous cytomegalovirus infection with a dramatic clinical presentation. J Am Acad Dermatol 1998; 38:349-51.

3. Rezaei N, Aghamohammadi A. Hyper-IgE syndrome. J Postgrad Med 2010; 56:63-4.

4. Engelhardt KR, McGhee S, Winkler S et al. Large deletions and point mutations involving the dedicator of cytokinesis 8 (DOCK8) in the autosomal-recessive form of hyper-IgE syndrome. J Allergy Clin Immunol 2009; 124:1289-302. 